\title{
Backward Stochastic $H_{2} / H_{\infty}$ Control: Infinite Horizon Case
}

\author{
Zhen $W^{1}$ and Qixia Zhang ${ }^{2}$ \\ ${ }^{1}$ School of Mathematics, Shandong University, Jinan 250100, China \\ ${ }^{2}$ School of Mathematical Sciences, University of Jinan, Jinan 250022, China \\ Correspondence should be addressed to Qixia Zhang; zhangqixial10@163.com
}

Received 4 March 2014; Accepted 1 May 2014; Published 22 May 2014

Academic Editor: Weihai Zhang

Copyright (C) 2014 Z. Wu and Q. Zhang. This is an open access article distributed under the Creative Commons Attribution License, which permits unrestricted use, distribution, and reproduction in any medium, provided the original work is properly cited.

The mixed $\mathrm{H}_{2} / \mathrm{H}_{\mathrm{\infty}}$ control problem is studied for systems governed by infinite horizon backward stochastic differential equations
(BSDEs) with exogenous disturbance signal. A necessary and sufficient condition for the existence of a unique solution to the
$\mathrm{H}_{2} / \mathrm{H}_{\mathrm{\infty}}$ control problem is derived. The equivalent feedback solution is also discussed. Contrary to deterministic or stochastic
forward case, the feedback solution is no longer feedback of the current state; rather, it is feedback of the entire history of the state.

\section{Introduction}

$H_{\infty}$ control is one of the most important robust control approaches in which control law is sought to efficiently eliminate the effect of the exogenous disturbance in the practical system. We refer the reader to [1-3] and the references therein. If the purpose is to select control not only to restrain the exogenous disturbance, but also to minimize a cost function when the worst case disturbance $d^{*}$ is implemented, this is the so-called mixed $H_{2} / H_{\infty}$ control problem. Mixed $H_{2} / H_{\infty}$ control problem has attracted much attention and has been widely applied to various fields. Please refer to [4, 5] for more information.

It should be pointed out that the above-mentioned works are concerned only with the forward stochastic systems. The case of systems governed by backward stochastic differential equations with exogenous disturbance signal, to our best knowledge, seems to be open. The objective of this paper is to develop an $\mathrm{H}_{2} / \mathrm{H}_{\infty}$ control theory for infinite horizon backward stochastic systems.

A BSDE is an Itô stochastic differential equation (SDE) for which a random terminal condition on the state has been specified. Since BSDEs are well-defined dynamic systems, it is very natural and appealing to study the control problems involving BSDEs as well as their applications in lots of different fields, especially in finance, economics, insurance, and so forth. Please refer to [6-12] for more details. This paper is concerned with mixed $\mathrm{H}_{2} / \mathrm{H}_{\infty}$ control of backward systems governed by infinite horizon linear BSDEs, namely, an infinite horizon backward stochastic $H_{2} / H_{\infty}$ control problem. This means that our purpose is to study mixed $H_{2} / H_{\infty}$ backward stochastic control problem in infinite horizon which presents more robust and stable sense in practise. For that, as preliminaries, we first need to review some results on infinite horizon BSDEs in Section 2. Chen and Wang [13] gave an existence and uniqueness result under a kind of Lipschitz condition suitable for one-dimensional infinite horizon BSDEs. Wu [14] generalized the result of [13] into the poisson jump process case in unbounded stopping time duration and obtained the corresponding comparison theorem. In this section, under this frame, we get the existence and uniqueness result for the infinite horizon matrix-valued BSDEs.

In Section 3, similar to the deterministic or stochastic forward case, we formulate the infinite horizon backward stochastic $H_{2} / H_{\infty}$ control problem. In Section 4 , a necessary and sufficient condition for the existence of a unique solution to the $\mathrm{H}_{2} / \mathrm{H}_{\infty}$ control problem is derived. It is shown that the existence of a unique solution to the control problem is equivalent to the corresponding uncontrolled perturbed system to have a $\mathbb{L}_{2}$-gain less than or equal to $\gamma$ and the resulting solution is characterized by the solution of an uncontrolled forward backward stochastic differential equation (FBSDE). 
Under some monotone assumptions, Hu and Peng [15] and Peng and $\mathrm{Wu}$ [16] obtained the existence and uniqueness results in an arbitrarily prescribed time duration. Wu and $\mathrm{Xu}$ [17] gave some comparison theorems for FBSDEs. Riccati equation plays an important role to get the feedback form of the optimal control; please refer to Yong and Zhou [18] for the details. Section 5 gives the equivalent linear feedback solution by virtue of the solution of a Riccati-type equation. As it turns out, the infinite horizon backward stochastic $\mathrm{H}_{2} / \mathrm{H}_{\infty}$ control can no longer be expressed as a linear feedback of the current state like that in deterministic or stochastic forward case. Rather, it depends, in general, on the entire past history of the state pair $(x(\cdot), z(\cdot))$.

\section{Notations and Preliminary Results of Infinite Horizon BSDEs}

To treat the infinite horizon backward stochastic $H_{2} / H_{\infty}$ control problem, we need the following preliminary results of infinite horizon BSDEs.

Let $\left(\Omega, \mathscr{F}_{,} \mathscr{F}_{t}, P\right)$ be a completed filtering probability space; let $\left(W_{t}\right)_{t \geq 0}$ be a standard one-dimensional Wiener process (our assumption that $W(\cdot)$ is scalar-valued is for the sake of simplicity; no essential difficulties are encountered when extending our analysis to the case of vector-valued Wiener process). $\left\{\mathscr{F}_{t}\right\}_{t \geq 0}$ is the natural filtration generated by this Wiener process $W(\cdot)$ up to time $t$, where $\mathscr{F}_{0}$ contains all $P$-null sets of $\mathscr{F}$ and $\mathscr{F}_{\infty}=\vee_{t \geq 0} \mathscr{F}_{t}$.

Throughout this paper, we adopt the following conventional notations. $S^{n}$ : the set of symmetric $n \times n$ matrices with real elements; $A^{T}$ : the transpose of the matrix $A$; $A \geq 0(A>0): A$ is positive semidefinite (positive definite) real matrix; $I$ : identity matrix; $\|x\|:=x^{T} x=$ $\left(\sum_{i=1}^{n}\left|x_{i}\right|^{2}\right)^{1 / 2}$ for $n$-dimensional vector $x=\left(x_{1}, \ldots, x_{n}\right)^{T}$; $\|A\|:=\max _{x \in R^{n},\|x\|=1}\|A x\|$ for $A \in R^{n \times n} ; N(\cdot)>(\geq 0): N(t)>$ $(\geq) 0$ for a.s. $t \in R^{+} ; M(\cdot)>(\geq) N(\cdot): M(\cdot)-N(\cdot)>(\geq) 0 ; X: \mathrm{a}$ given Hilbert space;

$$
\begin{aligned}
& L_{\mathscr{F}}^{2}\left(R^{+} ; X\right) \\
& =:\left\{f: R^{+} \times \Omega \longrightarrow X \text { is an } \mathscr{F}_{t}\right. \\
& \text { - adapted process such that } \mathbb{E} \int_{0}^{\infty}\|f(t)\|^{2} d t \\
& <\infty\} \\
& \mathcal{S}^{2}=:\left\{v_{t}, 0 \leq t \leq \infty, \text { is an } \mathscr{F}_{t}\right. \\
& \text { - adapted process such that } \mathbb{E}\left[\sup _{0 \leq t \leq \infty}\left\|v_{t}\right\|^{2}\right] \\
& <\infty\}
\end{aligned}
$$

$$
\begin{aligned}
L^{2}=:\{\xi & \xi \text { is a vector-valued } \mathscr{F}_{\infty} \\
& - \text { measurable random variable such that } \mathbb{E}\|\xi\|^{2} \\
& <\infty\} .
\end{aligned}
$$

We consider the infinite horizon BSDE:

$$
x_{t}=\xi+\int_{t}^{\infty} f\left(s, x_{s}, z_{s}\right) d s-\int_{t}^{\infty} z_{s} d W_{s}, \quad t \in[0, \infty]
$$

$(x, z)$ take value in $R^{n} \times R^{n}, \xi \in L^{2}$, and $f$ is a map from $\Omega \times[0, \infty] \times R^{n} \times R^{n}$ onto $R^{n}$ which satisfies the following.

(H2.1) For all $(x, z) \in R^{n} \times R^{n}, f(\cdot, x, z)$ is progressively measurable and

$$
\mathbb{E}\left(\int_{0}^{\infty}\|f(s, 0,0)\| d s\right)^{2}<\infty
$$

(H2.2) There exist two positive deterministic functions $u_{1}(t)$ and $u_{2}(t)$ such that, for all $\left(x_{i}, z_{i}\right) \in R^{n} \times R^{n}, i=1,2$,

$$
\begin{aligned}
& \left\|f\left(t, x_{1}, z_{1}\right)-f\left(t, x_{2}, z_{2}\right)\right\| \\
& \leq u_{1}(t)\left\|x_{1}-x_{2}\right\|+u_{2}(t)\left\|z_{1}-z_{2}\right\|, \quad t \in[0, \infty), \\
& \quad \int_{0}^{\infty} u_{1}(t) d t<\infty, \quad \int_{0}^{\infty} u_{2}^{2}(t) d t<\infty .
\end{aligned}
$$

Then we have the following.

Theorem 1 (see $\mathrm{Wu}[14])$. There exists a unique solution $(x, z) \in \mathcal{S}^{2} \times L_{\mathscr{F}}^{2}\left(R^{+} ; R^{n}\right)$ satisfying the BSDE (2).

Let us again consider a function $F$, which will be in the sequel the generator of the BSDE, defined on $\Omega \times[0, \infty] \times S^{n} \times S^{n}$, with values in $S^{n}$, such that the process $(F(t, y, z))_{t \in[0, \infty]}$ is a progressively measurable process for each $(y, z) \in S^{n} \times S^{n}$.

Along the line of Chen and Wang [13] or Wu [14] combined with that in Peng [19] for matrixed-valued BSDEs result in finite horizon, we get the following existence and uniqueness theorem for infinite horizon matrix-valued BSDEs.

Theorem 2. Suppose that $F$ satisfies the following.

$\left(\mathrm{H} 2.1^{\prime}\right)$ For all $(y, z) \in S^{n} \times S^{n}, F(\cdot, y, z)$ is progressively measurable and

$$
\mathbb{E}\left(\int_{0}^{\infty}\|F(s, 0,0)\| d s\right)^{2}<\infty
$$

$\left(\mathrm{H} 2.2^{\prime}\right)$ There exist two positive deterministic functions $u_{1}(t)$ and $u_{2}(t)$ such that, for all $\left(y_{i}, z_{i}\right) \in R^{n} \times R^{n}, i=1,2$,

$$
\begin{aligned}
& \left\|F\left(t, y_{1}, z_{1}\right)-F\left(t, y_{2}, z_{2}\right)\right\| \\
& \quad \leq u_{1}(t)\left\|y_{1}-y_{2}\right\|+u_{2}(t)\left\|z_{1}-z_{2}\right\|, \quad t \in[0, \infty),
\end{aligned}
$$


and $\int_{0}^{\infty} u_{1}(t) d t<\infty, \int_{0}^{\infty} u_{2}^{2}(t) d t<\infty, \xi$ is a given $S^{n}$-valued random variable, and $\xi \in L^{2}$. Then, the following matrixvalued infinite horizon BSDE

$$
Y_{t}=\xi+\int_{t}^{\infty} F\left(s, Y_{s}, Z_{s}\right) d s-\int_{t}^{\infty} Z_{s} d W_{s}
$$

admits a unique solution $(Y, Z) \in \mathcal{S}^{2} \times L_{\mathscr{F}}^{2}\left(R^{+} ; R^{n}\right)$.

\section{Problem Statement}

Now, we consider the following stochastic control system governed by an infinite horizon linear BSDE:

$$
\begin{aligned}
x(t)=\xi-\int_{t}^{\infty}[A(s) x(s)+B(s) u(s) \\
\quad+C(s) d(s)+D(s) z(s)] d s \\
-\int_{t}^{\infty} z(s) d W(s) .
\end{aligned}
$$

$Z \in \mathscr{R}^{n_{Z}}$ is the penalty output, and the energy of the output signal $Z$ is given by

$$
\|Z\|_{2}^{2}=x_{0}^{T} H x_{0}+\mathbb{E} \int_{0}^{\infty}\left[x_{t}^{T} Q_{t} x_{t}+z_{t}^{T} S_{t} z_{t}+u_{t}^{T} u_{t}\right] d t
$$

where $H$ is a nonnegative symmetric constant matrix and $Q_{t}(\omega)$ and $S_{t}(\omega)$ are nonnegative symmetric bounded progressively measurable matrix-valued processes. $u$ and $d$ stand for the control input and exogenous disturbance signal, respectively. The energy of the disturbances is

$$
\|d\|_{2}^{2}=\mathbb{E} \int_{0}^{\infty} d_{t}^{T} d_{t} d t
$$

Later, we will state assumptions on the coefficients $A(\cdot)$, $B(\cdot), C(\cdot), D(\cdot), Q(\cdot), S(\cdot)$ so as to guarantee the existence of a unique solution pair $(x(\cdot), z(\cdot)) \in \mathcal{S}^{2} \times L_{\mathscr{F}}^{2}\left(R^{+} ; R^{n}\right)$ of BSDE (8) for any $u \in L_{\mathscr{F}}^{2}\left(R^{+} ; R^{n_{u}}\right), d \in L_{\mathscr{F}}^{2}\left(R^{+} ; R^{n_{d}}\right)$, and $\xi \in L^{2}$. We refer to such a four-tuple $(x(\cdot), z(\cdot) ; u(\cdot), d(\cdot))$ as an admissible triple.

Now, we first define the infinite horizon backward stochastic $H_{2} / H_{\infty}$ control as follows.

Definition 3 (backward stochastic $H_{2} / H_{\infty}$ control). For given $\gamma>0$ and $d \in L_{\mathscr{F}}^{2}\left(R^{+} ; R^{n_{d}}\right)$, find, if possible, a control $u=$ $u^{*} \in L_{\mathscr{F}}^{2}\left(R^{+} ; R^{n_{u}}\right)$, such that

(i) the trajectory of the closed-loop system (8) with $\xi=0$ satisfies

$$
\|Z\|_{2}^{2} \leq \gamma^{2}\|d\|_{2}^{2}, \quad \forall d \neq 0 \in L_{\mathscr{F}}^{2}\left(R^{+} ; R^{n_{d}}\right) \text { and }
$$

(ii) when the worst case disturbance ([4]) $d^{*} \in$ $L_{\mathscr{F}}^{2}\left(R^{+} ; R^{n_{d}}\right)$, if existing, is implemented in (8), $u^{*}$ minimizes the quadratic performance $\|Z\|_{2}^{2}$ simultaneously.
If we define

$$
\begin{aligned}
& J_{1}(u, d)=\|Z\|_{2}^{2}-\gamma^{2}\|d\|_{2}^{2}, \\
& J_{2}(u, d)=\|Z\|_{2}^{2}
\end{aligned}
$$

then the mixed $H_{2} / H_{\infty}$ control problem is equivalent to find the Nash equilibria $\left(u^{*}, d^{*}\right)$ defined as

$$
\begin{gathered}
J_{1}\left(u^{*}, d^{*}\right) \geq J_{1}\left(u^{*}, d\right), \quad \forall d \in L_{\mathscr{F}}^{2}\left(R^{+} ; R^{n_{d}}\right), \\
J_{2}\left(u, d^{*}\right) \geq J_{2}\left(u^{*}, d^{*}\right), \quad \forall u \in L_{\mathscr{F}}^{2}\left(R^{+} ; R^{n_{u}}\right), \\
J_{1}\left(u^{*}, d\right) \leq 0, \quad \forall d \neq 0 \in L_{\mathscr{F}}^{2}\left(R^{+} ; R^{n_{d}}\right), \quad \xi=0 .
\end{gathered}
$$

Obviously, inequality (15) is associated with the $H_{\infty}$ performance. The first Nash inequality (13) is to keep that $d^{*}$ is the worst case disturbance, while the second one (14) is related with the $\mathrm{H}_{2}$ performance. Clearly, if the Nash equilibria $\left(u^{*}, d^{*}\right)$ exist and satisfy inequality (15), then $u^{*}$ is our desired $H_{2} / H_{\infty}$ controller and $d^{*}$ is the worst case disturbance. In this case, we also say that the infinite horizon backward stochastic $\mathrm{H}_{2} / \mathrm{H}_{\infty}$ control admits a solution $\left(u^{*}, d^{*}\right)$.

Throughout this paper, we assume the following.

(A1) All matrices mentioned in this paper are bounded progressively measurable processes.

(A2)

$$
\begin{aligned}
& \mathbb{E} \int_{0}^{\infty}\|A(t)\| d t<\infty, \\
& \mathbb{E} \int_{0}^{\infty}\|D(t)\|^{2} d t<\infty .
\end{aligned}
$$

(A3)

$$
\begin{gathered}
\gamma>0, \\
Q \geq 0, \quad \mathbb{E} \int_{0}^{\infty}\|Q(t)\| d t<\infty, \\
B B^{T}(\cdot)>\frac{C C^{T}(\cdot)}{\gamma^{2}}, \\
\mathbb{E} \int_{0}^{\infty}\left\|B(t) B(t)^{T}-\frac{C(t) C(t)^{T}}{\gamma^{2}}\right\| d t<\infty .
\end{gathered}
$$

From Theorem 2, we obtain that assumption (A2) is sufficient to guarantee the existence of a unique solution pair $(x(\cdot), z(\cdot)) \in \mathcal{S}^{2} \times L_{\mathscr{F}}^{2}\left(R^{+} ; R^{n}\right)$ of BSDE (8) for any $u \in$ $L_{\mathscr{F}}^{2}\left(R^{+} ; R^{n_{u}}\right)$ and $d \in L_{\mathscr{F}}^{2}\left(R^{+} ; R^{n_{d}}\right)$.

\section{The Necessary and Sufficient Condition}

In this section, we give a necessary and sufficient condition for the existence of a unique solution to the backward stochastic $H_{2} / H_{\infty}$ control problem. We begin our presentation with some preliminaries. 
Consider the following uncontrolled stochastic perturbed system:

$$
\begin{array}{r}
d x_{t}=\left[A(t) x_{t}+C(t) d_{t}+D(t) z_{t}\right] d t+z_{t} d B(t), \\
x(\infty)=\xi, \quad t \in[0, \infty) .
\end{array}
$$

Let $Z$ be the to-be-controlled output. For any $0<T<$ $\infty$, define the perturbation operator $\mathbb{L}: L_{\mathscr{F}}^{2}\left(R^{+} ; R^{n_{d}}\right) \rightarrow$ $L_{\mathscr{F}}^{2}\left(R^{+} ; R^{n_{Z}}\right)$ as

$$
\mathbb{L}(d)=\left.Z\right|_{x_{\infty}=0}, \quad t \geq 0, d \in L_{\mathscr{F}}^{2}\left(R^{+} ; R^{n_{d}}\right),
$$

with its norm

$$
\begin{aligned}
\|\mathbb{L}\|_{2} & :=\sup _{d \in L_{\mathscr{F}}^{2}\left(R^{+} ; R^{n} d\right), d \neq 0, x_{\infty}=0} \frac{\|\mathbb{L}(d)\|_{2}}{\|d\|_{2}} \\
& =\sup _{d \in L_{\mathscr{F}}^{2}\left(R^{+} ; R^{n} d\right), d \neq 0, x_{\infty}=0} \frac{\|Z\|_{2}}{\|d\|_{2}},
\end{aligned}
$$

where

$$
\|Z\|_{2}^{2}=x_{0}^{T} H x_{0}+\mathbb{E} \int_{0}^{\infty}\left[x_{t}^{T} Q_{t} x_{t}+y_{t}^{T} S_{t} y_{t}\right] d t .
$$

Obviously, $\mathbb{L}$ is a nonlinear operator.

Definition 4. Let $\gamma>0$; system (18) is said to have $\mathbb{L}_{2}$-gain less than or equal to $\gamma$ if for any nonzero $d \in L_{\mathscr{F}}^{2}\left(R^{+} ; R^{n_{d}}\right)$, $\|\mathbb{L}\|_{2} \leq \gamma$.

Proposition 5. For system (18) and given disturbance attenuation $\gamma>0$, if there exists a function $\mathbf{P}(\cdot)$, satisfying the following SDE (the variables $t$ and $\omega$ are suppressed):

$$
\begin{gathered}
d P=\left[-A^{T} P-P A-Q-\frac{P C C^{T} P}{\gamma^{2}}\right] d t-D^{T} P d W(t), \\
P+S \leq 0, \quad P(0)=-H, \quad t \in[0, \infty),
\end{gathered}
$$

then $\|\mathbb{L}\|_{2} \leq \gamma$.

Proof. It only needs to note the following identity:

$$
\begin{aligned}
& \|Z\|_{2}^{2}-\gamma^{2}\|d\|_{2}^{2} \\
& =\|Z\|_{2}^{2}-\gamma^{2}\|d\|_{2}^{2}+\mathbb{E} \int_{0}^{\infty} d\left(x^{T} P x\right)-x_{0}^{T} H x_{0} \\
& =-\gamma^{2}\left\|v-\frac{C^{T} P x}{\gamma^{2}}\right\|_{2}^{2}+\mathbb{E} \int_{0}^{\infty} y^{T}(P+S) y d t \leq 0 .
\end{aligned}
$$

The following theorem is a necessary and sufficient condition for the existence of a unique solution to the infinite horizon backward stochastic $\mathrm{H}_{2} / \mathrm{H}_{\infty}$ control problem.

Theorem 6. For system (8), the backward stochastic $\mathrm{H}_{2} / \mathrm{H}_{\infty}$ control problem admits a solution if and only if the corresponding uncontrolled system (18) has $\mathbb{L}_{2}$-gain less than or equal to $\gamma$.
Moreover, if the backward stochastic $\mathrm{H}_{2} / \mathrm{H}_{\infty}$ control problem admits a solution, then the solution is unique with

$$
u^{*}=\frac{B^{T} p^{*}}{2}, \quad d^{*}=-\frac{C^{T} p^{*}}{2 \gamma^{2}}
$$

where $\left(p^{*}, x^{*}, z^{*}\right)$ is the solution of the following FBSDE:

$$
\begin{gathered}
d p_{t}^{*}=\left[2 Q x_{t}^{*}-A^{T} p_{t}^{*}\right] d t+\left[2 S z_{t}^{*}-D^{T} p_{t}^{*}\right] d B(t), \\
d x_{t}^{*}=\left[A x_{t}^{*}+\frac{B B^{T} p_{t}^{*}}{2}-\frac{C C^{T} p_{t}^{*}}{2 \gamma^{2}}+D z_{t}^{*}\right] d t+z_{t}^{*} d B(t), \\
p_{0}^{*}=2 H x_{0}^{*}, \quad x_{\infty}^{*}=\xi, \quad t \in[0, \infty) .
\end{gathered}
$$

Proof. (1) The Sufficient Condition. To show that the backward stochastic $\mathrm{H}_{2} / \mathrm{H}_{\infty}$ control problem admits a unique solution $\left(u^{*}, d^{*}\right)$ if the corresponding uncontrolled system (18) has $\mathbb{L}_{2}$ gain less than or equal to $\gamma$, we will show that $\left(u^{*}, d^{*}\right)$ is a solution firstly.

Look at the above FBSDE; from [16], the FBSDE (25) has a unique solution $\left(p_{t}^{*}, x_{t}^{*}, z_{t}^{*}\right)$. Now, we try to prove that $d^{*}$ is the worst case disturbance. For any given $d \in L_{\mathscr{F}}^{2}\left(R^{+} ; R^{n_{d}}\right)$, suppose that $x^{d}$ is the trajectory corresponding to $\left(u^{*}, d\right) \epsilon$ $L_{\mathscr{F}}^{2}\left(R^{+} ; R^{n_{u}}\right) \times L_{\mathscr{F}}^{2}\left(R^{+} ; R^{n_{d}}\right)$. It is easy to see the trajectory corresponding to

$$
\left(0, d^{*}-d\right)
$$

is $x^{*}-x^{d}$ with initial state $x_{0}^{*}-x_{0}^{d}$ and terminal state 0 . Hence, $\left(x^{*}-x^{d}, z^{*}-z^{d}\right)$ is the solution corresponding to $d^{*}-d$ for system (18) with terminal state 0 . Since system (18) has $\mathbb{L}_{2}{ }^{-}$ gain less than or equal to $\gamma$, then

$$
\begin{gathered}
\mathbb{E}\left[\int _ { 0 } ^ { \infty } \left[-\left(x^{*}-x^{d}\right)^{T} Q\left(x^{*}-x^{d}\right)-\left(z^{*}-z^{d}\right)^{T} S\left(z^{*}-z^{d}\right)\right.\right. \\
\left.\left.+\gamma^{2}\left(d^{*}-d\right)^{T}\left(d^{*}-d\right)\right] d t\right] \\
\quad-\left(x_{0}^{*}-x_{0}^{d}\right)^{T} H\left(x_{0}^{*}-x_{0}^{d}\right) \geq 0 \\
J_{2}\left(u^{*}, d^{*}\right)-J_{2}\left(u^{*}, d\right) \\
=\mathbb{E}\left[\int _ { 0 } ^ { \infty } \left[x^{* T} Q x^{*}-x^{d T} Q x^{d}+z^{* T} S z^{*}-z^{d T} S z^{d}\right.\right. \\
\left.\left.\quad-\gamma^{2} d^{* T} d^{*}+\gamma^{2} d^{T} d\right] d t\right] \\
+x_{0}^{* T} H x_{0}^{*}-x_{0}^{d T} H x_{0}^{d}
\end{gathered}
$$




$$
\begin{gathered}
=\mathbb{E}\left[\int _ { 0 } ^ { \infty } \left[\left(x^{*}-x^{d}\right)^{T} \mathrm{Q}\left(x^{*}-x^{d}\right)\right.\right. \\
\quad-2 x^{d T} \mathrm{Q}\left(x^{d}-x^{*}\right)+\left(z^{*}-z^{d}\right)^{T} S\left(z^{*}-z^{d}\right) \\
\left.\left.\quad-2 z^{d T} S\left(z^{d}-z^{*}\right)-\gamma^{2} d^{* T} d^{*}+\gamma^{2} d^{T} d\right] d t\right] \\
+\left(x_{0}^{*}-x_{0}^{d}\right)^{T} H\left(x_{0}^{*}-x_{0}^{d}\right)-2 x_{0}^{d T} H\left(x_{0}^{d}-x_{0}^{*}\right) .
\end{gathered}
$$

Applying Itô's formula to $p^{* T}\left(x^{d}-x^{*}\right)$,

$$
\begin{aligned}
& -2 x_{0}^{* T} H\left(x_{0}^{d}-x_{0}^{*}\right) \\
& =\mathbb{E}\left[\int_{0}^{\infty} d\left[p^{* T}\left(x^{d}-x^{*}\right)\right]\right. \\
& =\mathbb{E} \int_{0}^{\infty}\left[2 x^{* T} Q\left(x^{d}-x^{*}\right)\right. \\
& \left.\left.\quad+2 z^{* T} S\left(z^{d}-z^{*}\right)+\left(C^{T} p^{*}\right)^{T}\left(d-d^{*}\right)\right] d t\right] \\
& =\mathbb{E}\left[\int _ { 0 } ^ { \infty } \left[2 x^{* T} Q\left(x^{d}-x^{*}\right)\right.\right. \\
& \left.\left.+2 z^{* T} S\left(z^{d}-z^{*}\right)-2 \gamma^{2} v^{* T}\left(d-d^{*}\right)\right] d t\right] .
\end{aligned}
$$

Substituting $2 x_{0}^{* T} H\left(x_{0}^{d}-x_{0}^{*}\right)$ into (28), we get

$$
\begin{gathered}
J_{2}\left(u^{*}, d^{*}\right)-J_{2}\left(u^{*}, d\right) \\
=\mathbb{E}\left[\int _ { 0 } ^ { \infty } \left[-\left(x^{*}-x^{d}\right)^{T} \mathrm{Q}\left(x^{*}-x^{d}\right)\right.\right. \\
-\left(z^{*}-z^{d}\right)^{T} S\left(z^{*}-z^{d}\right) \\
\left.\left.+\gamma^{2}\left(d^{*}-d\right)^{T}\left(d^{*}-d\right)\right] d t\right] \\
-\left(x_{0}^{*}-x_{0}^{d}\right)^{T} H\left(x_{0}^{*}-x_{0}^{d}\right) .
\end{gathered}
$$

From (27), then

$$
J_{2}\left(u^{*}, d^{*}\right)-J_{2}\left(u^{*}, d\right) \geq 0 .
$$

So $d^{*}$ is the worst case disturbance. Moreover, for $x_{\infty}=0$, the FBSDE (25) admits a unique solution $\left(p^{*}, x^{*}, z^{*}\right)=(0,0,0)$; then

$$
J_{2}\left(u^{*}, d\right) \leq J_{2}\left(u^{*}, d^{*}\right)=0 .
$$

Hence, $u^{*}$ restrains the exogenous disturbance. In the following, we will show that $u^{*}$ also minimizes that cost function when the worst case disturbance $d^{*}$ is implemented into system (8).

For any $u \in L_{\mathscr{F}}^{2}\left(\mathbb{R}^{n_{u}}\right)$, let $x_{t}^{u}$ be the trajectory of the system (8) corresponding to $\left(u, d^{*}\right)$. Let us first consider

$$
J_{1}\left(u^{*}, d^{*}\right)-J_{1}\left(u, d^{*}\right)=I_{1},
$$

where

$$
\begin{aligned}
& I_{1}=-\mathbb{E}\left[\int _ { 0 } ^ { \infty } \left[x^{* T} Q(t) x^{*}-x^{u T} Q x^{u}+z^{* T} S(t) z^{*}\right.\right. \\
& \left.\left.\quad-z^{u T} S z^{u}+u^{* T} u^{*}-u^{T} u\right] d t\right] \\
& +x_{0}^{* T} H x_{0}^{*}-x_{0}^{u T} H x_{0}^{u} \\
& =\mathbb{E}\left[\int _ { 0 } ^ { \infty } \left[\left(x^{*}-x^{u}\right)^{T} Q\left(x^{*}-x^{u}\right)\right.\right. \\
& \quad+\left(z^{*}-z^{u}\right)^{T} S\left(z^{*}-z^{u}\right)+\left(u^{*}-u\right)^{T}\left(u^{*}-u\right) \\
& +2 x^{* T} Q(t)\left(x^{u}-x^{*}\right)+2 y^{* T} S(t)\left(z^{u}-z^{*}\right) \\
& \left.\left.+2 u^{* T}\left(u-u^{*}\right)\right] d t\right] \\
& +\left(x_{0}^{*}-x_{0}^{u}\right)^{T} H\left(x_{0}^{*}-x_{0}^{u}\right)+2 x_{0}^{* T} H\left(x_{0}^{u}-x_{0}^{*}\right) .
\end{aligned}
$$

From $p_{0}^{*}=2 H x_{0}^{*}$, we use Itô's formula to $p_{t}^{* T}\left(x_{t}^{u}-x_{t}^{*}\right)$ and get

$$
\begin{aligned}
& 2 x_{0}^{* T} H\left(x_{0}^{u}-x_{0}^{*}\right) \\
& =-\mathbb{E}\left[\int _ { 0 } ^ { \infty } \left[2 x^{* T} Q(t)\left(x^{u}-x^{*}\right)\right.\right. \\
& \left.\left.\quad+2 z^{* T} S\left(z^{u}-z^{*}\right)+2 u^{* T}\left(u-u^{*}\right)\right] d t\right] .
\end{aligned}
$$

Then because of $Q, S$, and $H$ being nonnegative, we have

$$
J_{1}\left(u^{*}, v^{*}\right)-J_{1}\left(u, v^{*}\right)=I_{1} \geq 0 .
$$

Therefore, $d^{*}$ minimizes the cost function when the worst case disturbance $d^{*}$ is implemented into system (8).

So, $\left(u^{*}, d^{*}\right)=\left(B^{T} p^{*} / 2,-C^{T} p^{*} / 2 \gamma^{2}\right)$ is a solution of the backward stochastic $H_{2} / H_{\infty}$ control problem.

We are now in a position to prove the uniqueness of the solution. Assume that the backward stochastic $H_{2} / H_{\infty}$ control has a solution $\left(u^{1}, d^{1}\right),\left(x^{1}, z^{1}\right)$ is the corresponding solution for (8), and $p^{1}$ is the solution of the following BSDE:

$$
\begin{aligned}
& d p^{1}=\left[2 Q x^{1}-A^{T} p^{1}\right] d t+\left[2 S z^{1}-D^{T} p^{1}\right] d B(t), \\
& p_{0}^{1}=2 H x_{0}^{1} .
\end{aligned}
$$

Implementing $d^{1}$, having

$$
\inf _{u \in L_{\mathscr{F}}^{2}\left(R^{n_{u}}\right)} J_{1}\left(u, d^{1}\right)
$$

is a standard LQ optimal control problem. By uniqueness, $u^{1}=B^{T} p^{1} / 2$. 
Let $x$ be the trajectory corresponding to $\left(u^{1}, d\right)=$ $\left(u^{1},-C^{T} p^{1} / \gamma^{2}\right)$; then

$$
\begin{aligned}
& 0 \geq J_{2}\left(u^{1}, d\right)-J_{2}\left(u^{1}, d^{1}\right) \\
& =\mathbb{E}\left[\int _ { 0 } ^ { \infty } \left[x^{T} Q x-x^{1 T} Q x^{1}+z^{T} S z-z^{1 T} S z^{1}\right.\right. \\
& \left.\left.-\gamma^{2} d^{T} d+\gamma^{2} d^{1 T} d^{1}\right] d t\right] \\
& +x_{0}^{T} H x_{0}-x_{0}^{1 T} H x_{0}^{1} \\
& =\mathbb{E}\left[\int _ { 0 } ^ { \infty } \left[\left(x^{1}-x\right)^{T} Q\left(x^{1}-x\right)\right.\right. \\
& -2 x^{1 T} Q\left(x^{1}-x\right)+\left(z^{1}-z\right)^{T} S\left(z^{1}-z\right) \\
& \left.\left.-2 z^{1 T} S\left(z^{1}-z\right)+\gamma^{2} d^{1 T} d^{1}-\gamma^{2} d^{T} d\right] d t\right] \\
& +\left(x_{0}^{1}-x_{0}\right)^{T} H\left(x_{0}^{1}-x_{0}\right)-2 x_{0}^{1 T} H\left(x_{0}^{1}-x_{0}\right) .
\end{aligned}
$$

Applying Itô's formula to $p^{1 T}\left(x^{1}-x\right)$,

$$
\begin{aligned}
& 2 x_{0}^{1 T} H\left(x_{0}^{1}-x_{0}\right) \\
& =\mathbb{E} \int_{0}^{\infty} d\left[p^{1 T}\left(x^{1}-x\right)\right] \\
& =\mathbb{E}\left[\int _ { 0 } ^ { \infty } \left[2 x^{1 T} Q\left(x^{1}-x\right)\right.\right. \\
& \left.\left.\quad+\left(C^{T} z^{1}\right)^{T}\left(d^{1}-d\right)+2 z^{1 T} S\left(z^{1}-z\right)\right] d t\right] \\
& =\mathbb{E}\left[\int _ { 0 } ^ { \infty } \left[2 x^{1 T} Q\left(x^{1}-x\right)\right.\right. \\
& \left.\left.\quad+2 z^{1 T} S\left(z^{1}-z\right)-2 \gamma^{2} d^{T}\left(d^{1}-d\right)\right] d t\right] .
\end{aligned}
$$

Substituting $-2 x_{0}^{1 T} H\left(x_{0}^{1}-x_{0}\right)$ into (39), then

$$
\begin{aligned}
0 \geq J_{2}\left(\gamma, x_{0} ; u^{1}, d\right)-J_{2}\left(\gamma, x_{0} ; u^{1}, d^{1}\right) \\
=\mathbb{E}\left[\int _ { 0 } ^ { \infty } \left[\left(x^{1}-x\right)^{T} Q\left(x^{1}-x\right)\right.\right. \\
+\left(z^{1}-z\right)^{T} S\left(z^{1}-z\right) \\
\left.\left.+\gamma^{2}\left(z-z^{1}\right)^{T}\left(z-z^{1}\right)\right] d t\right] .
\end{aligned}
$$

Because of $Q, S$, and $M$ being nonnegative, we get $d^{1}=v=$ $C^{T} z^{1} / \gamma^{2}$.

Therefore, $\left(u^{1}, d^{1}\right)=\left(u^{*}, d^{*}\right)$.

(2) The Necessary Condition. Here we assume that a solution exists; then from the uniqueness of the solution, we get that $\left(u^{*}, d^{*}\right)$ is the unique solution and we will show that system (18) has $\mathbb{L}_{2}$-gain less than or equal to $\gamma$.

For $x_{T}=0$, the $\operatorname{FBSDE}(25)$ has a unique solution $\left(p^{*}, x^{*}, z^{*}\right)=(0,0,0)$; then $\left(u^{*}, d^{*}\right)=(0,0)$ and

$$
J_{2}\left(u^{*}, d\right) \leq J_{2}\left(u^{*}, d^{*}\right)=0, \quad \forall d \in L_{\mathscr{F}}^{2}\left(\mathscr{R}^{n_{d}}\right)
$$

Therefore, system (18) has $\mathbb{L}_{2}$-gain less than or equal to $\gamma$.

\section{The Linear Feedback Solution}

The main result of this section gives the equivalent linear feedback solution. For the purpose of this section the coefficients $A_{t}, B_{t}, C_{t}, D_{t}, E_{t}, Q_{t}$, and $S_{t}$ are assumed deterministic functions; (18) has $\mathbb{L}_{2}$-gain less than or equal to $\gamma$.

Let $(p, x, z)$ be the solution of (25); we first give the relations between $p, x$, and $z$ using the undetermined coefficients method. Now, we introduce the following generalized matrixvalued Riccati equation (the variables $t$ are suppressed):

$$
\begin{aligned}
\dot{K} & -A K-K A^{T}+2 K Q K-\frac{B B^{T}}{2} \\
& +\frac{C C^{T}}{2 \gamma^{2}}+D(I-2 K S)^{-1} K D^{T}=0, \quad K(\infty)=0 .
\end{aligned}
$$

Similar to the line developed by Lim and Zhou [6], we can prove that (43) admits a unique solution $K(\cdot)$. Letting $K(\cdot)$ be the solution to (43), we define the following equations:

$$
\begin{aligned}
d h & =\left[A h-2 K Q h+D(I-2 K S)^{-1} \eta\right] d t+\eta d B(t), \\
h(\infty) & =\xi .
\end{aligned}
$$

Equation (44) is a linear BSDE and admits a unique solution $(h, \eta)$.

Theorem 7. Suppose that $(p(\cdot), x(\cdot), z(\cdot)), K(\cdot)$, and $(h(\cdot), \eta(\cdot))$ are the solutions of (25), (43), and (44), respectively; then the following relations are satisfied:

$$
\begin{aligned}
& x(t)=K(t) p(t)+h(t), \\
& z(t)=(I-2 K(t) S(t))^{-1}\left(\eta(t)-K(t) D(t)^{T} p(t)\right), \\
& x(0)=(I-2 K(0) H)^{-1} h(0) .
\end{aligned}
$$

Proof. Let $x(t)=K(t) p(t)+h(t)$. We apply Itô's formula to $x(t), K(t) p(t)+h(t)$, respectively, and it is easy to check that $K(t)$ and $h(t)$ satisfy (43) and (44), respectively.

From Theorem 7, we know that $x(\cdot)$ can be written to the functions of $K(\cdot), p(\cdot)$, and $h(\cdot)$. Now we would like to derive the feedback solution using the undetermined coefficients 
method. First, we introduce the generalized matrix-valued Riccati equation and a linear SDE:

$$
\begin{aligned}
& \dot{\Sigma}+\Sigma A+A^{T} \Sigma \\
& +\Sigma\left[\frac{B B^{T}}{2}-\frac{C C^{T}}{2 \gamma^{2}}-D(I-2 K S)^{-1} K D^{T}\right] \\
& \times \Sigma-2 Q=0, \\
& \Sigma(0)=2 H, \\
& d r \\
& \begin{aligned}
&= {\left[-A^{T} r-\frac{\Sigma B B^{T} r}{2}+\frac{\Sigma C C^{T} r}{2 \gamma^{2}}+\Sigma D(I-2 K S)^{-1} K D^{T} r\right.} \\
&\left.-\Sigma D(I-2 K S)^{-1} \eta\right] d t \\
&+ {\left[(2 S-\Sigma)(I-2 K S)^{-1}\left[\eta-K D^{T}(I-\Sigma K)^{-1}(\Sigma h+r)\right]\right.} \\
&\left.-D^{T}(I-\Sigma K)^{-1}(\Sigma h+r)\right] d B(t), \\
& r(0)=0 .
\end{aligned}
\end{aligned}
$$

Similar to the line developed by Lim and Zhou [6], we can prove that (46) admits a unique solution $\Sigma(\cdot)$. Equation (47) is a linear SDE and has a unique solution $r(\cdot)$.

Theorem 8. The backward stochastic $\mathrm{H}_{2} / \mathrm{H}_{\infty}$ control problem has a feedback solution $\left(u^{*}, v^{*}\right)$,

$$
u^{*}=\frac{B^{T}(\Sigma x+r)}{2}, \quad v^{*}=-\frac{C^{T}(\Sigma x+r)}{2 \gamma^{2}} .
$$

Proof. Let $p(t)=\Sigma(t) x(t)+r(t)$. We apply Itô's formula to $p(t)$ and $\Sigma(t) x(t)+r(t)$, respectively, and it is easy to check that $\Sigma(t)$ and $r(t)$ satisfy (46) and (47).

Remark 9. From Theorem 8, we see that the solution involves an additional random nonhomogeneous term $r(\cdot)$. This addition disqualifies (48) from a feedback control of the current state, contrary to the deterministic or stochastic forward $H_{2} / H_{\infty}$ (see $\left.[4,5]\right)$ cases. The reason is because $r(\cdot)$ depends on $(h(\cdot), \eta(\cdot))$, which in turn depends on $\xi$, the terminal condition of part of the state variable, $x(\cdot)$. This is one of the major distinctive features of the backward stochastic $\mathrm{H}_{2} / \mathrm{H}_{\infty}$ problem.

Finally, it is important to recognize that the expressions for the backward stochastic $H_{2} / H_{\infty}$ control, as presented in Theorems 6 and 8, are equivalent expressions of the same process; that is, this does not contradict the uniqueness of the solution.

We present an example to illustrate the above theoretical results as follows.
Example 10. Consider the backward stochastic $H_{2} / H_{\infty}$ control problem of the following one-dimensional system:

$x(t)$

$$
\begin{gathered}
=\xi-\int_{t}^{\infty}\left[2 e^{-s} x(s)+\sqrt{4 e^{-s}+2} u(s)\right. \\
\left.\quad+\sqrt{e^{-s}+1} d(s)+2 e^{-s / 2} z(s)\right] d s \\
-\int_{t}^{\infty} z(s) d W(s),
\end{gathered}
$$

with controlled output energy

$$
\|Z\|_{2}^{2}=\mathbb{E} \int_{0}^{\infty}\left[\frac{e^{-t} x_{t}^{2}}{2}+u_{t}^{2}\right] d t
$$

If we take $\gamma=\sqrt{2} / 2$, then the Riccati equation (43) specializes to

$$
K(t)=\int_{t}^{\infty}\left[e^{-s}\left(K(s)^{2}-1\right)\right] d s .
$$

Solving it yields $K(t)=\left(1-e^{2 e^{-t}}\right) /\left(1+e^{2 e^{-t}}\right)$. Equation (44) specializes to

$h(t)$

$$
\begin{aligned}
= & \xi-\int_{t}^{\infty}\left[\left\{2 e^{-s}-e^{-s} \frac{1-e^{2 e^{-s}}}{1+e^{2 e^{-s}}}\right\} h(s)+2 e^{-s / 2} \eta(t)\right] d t \\
& -\int_{t}^{\infty} \eta(t) d W(t) .
\end{aligned}
$$

Then, from Theorem 7, we get a unique solution

$$
\begin{aligned}
& \left(u^{*}, d^{*}\right) \\
& =\left(\sqrt{\frac{2 e^{-t}+1}{2}} \cdot \frac{1+e^{2 e^{-t}}}{1-e^{2 e^{-t}}} \cdot[x(t)-h(t)],\right. \\
& \left.\quad-\sqrt{e^{-t}+1} \cdot \frac{1+e^{2 e^{-t}}}{1-e^{2 e^{-t}}} \cdot[x(t)-h(t)]\right),
\end{aligned}
$$

of the backward $H_{2} / H_{\infty}$ control problem.

\section{Conflict of Interests}

The authors declare that there is no conflict of interests regarding the publication of this paper.

\section{Acknowledgments}

This work was supported by the National Natural Science Foundation (11221061 and 61174092), 111 project (B12023), the National Science Fund for Distinguished Young Scholars of China (no. 11125102), the Science and Technology Project of Shandong Province (2013GRC32201), and the Doctoral Foundation of University of Jinan (XBS1213). 


\section{References}

[1] J. C. Doyle, K. Glover, P. P. Khargonekar, and B. A. Francis, "State-space solutions to standard $\mathrm{H}_{2}$ and $H_{\infty}$ control problems," Institute of Electrical and Electronics Engineers. Transactions on Automatic Control, vol. 34, no. 8, pp. 831-847, 1989.

[2] D. Hinrichsen and A. J. Pritchard, "Stochastic $H_{\infty}$ ", SIAM Journal on Control and Optimization, vol. 36, no. 5, pp. 15041538, 1998.

[3] G. Zames, "Feedback and optimal sensitivity: model reference transformations, multiplicative seminorms, and approximate inverses," Institute of Electrical and Electronics Engineers. Transactions on Automatic Control, vol. 26, no. 2, pp. 301-320, 1981.

[4] B.-S. Chen and W. Zhang, "Stochastic $\mathrm{H}_{2} / \mathrm{H}_{\infty}$ control with state-dependent noise," Institute of Electrical and Electronics Engineers. Transactions on Automatic Control, vol. 49, no. 1, pp. 45-57, 2004.

[5] D. J. N. Limebeer, B. D. O. Anderson, and B. Hendel, "A Nash game approach to mixed $\mathrm{H}_{2} / \mathrm{H}_{\infty}$ control," Institute of Electrical and Electronics Engineers. Transactions on Automatic Control, vol. 39, no. 1, pp. 69-82, 1994.

[6] A. E. B. Lim and X. Y. Zhou, "Linear-quadratic control of backward stochastic differential equations," SIAM Journal on Control and Optimization, vol. 40, no. 2, pp. 450-474, 2001.

[7] J.-M. Bismut, "An introductory approach to duality in optimal stochastic control," SIAM Review, vol. 20, no. 1, pp. 62-78, 1978.

[8] D. Duffie and L. G. Epstein, "Stochastic differential utility," Econometrica, vol. 60, no. 2, pp. 353-394, 1992.

[9] N. El Karoui, S. Peng, and M. C. Quenez, "Backward stochastic differential equations in finance," Mathematical Finance, vol. 7, no. 1, pp. 1-71, 1997.

[10] É. Pardoux and S. G. Peng, "Adapted solution of a backward stochastic differential equation," Systems \& Control Letters, vol. 14, no. 1, pp. 55-61, 1990.

[11] G. Wang and Z. Yu, "A Pontryagin's maximum principle for non-zero sum differential games of BSDEs with applications," Institute of Electrical and Electronics Engineers. Transactions on Automatic Control, vol. 55, no. 7, pp. 1742-1747, 2010.

[12] G. Wang and Z. Yu, "A partial information non-zero sum differential game of backward stochastic differential equations with applications," Automatica, vol. 48, no. 2, pp. 342-352, 2012.

[13] Z. Chen and B. Wang, "Infinite time interval BSDEs and the convergence of $g$-martingales," Australian Mathematical Society. Journal A: Pure Mathematics and Statistics, vol. 69, no. 2, pp. 187-211, 2000.

[14] Z. Wu, "Fully coupled FBSDE with Brownian motion and Poisson process in stopping time duration," Journal of the Australian Mathematical Society, vol. 74, no. 2, pp. 249-266, 2003.

[15] Y. Hu and S. Peng, "Solution of forward-backward stochastic differential equations," Probability Theory and Related Fields, vol. 103, no. 2, pp. 273-283, 1995.

[16] S. Peng and Z. Wu, "Fully coupled forward-backward stochastic differential equations and applications to optimal control," SIAM Journal on Control and Optimization, vol. 37, no. 3, pp. 825-843, 1999.

[17] Z. Wu and M. Xu, "Comparison theorems for forward backward SDEs," Statistics \& Probability Letters, vol. 79, no. 4, pp. 426-435, 2009.

[18] J. Yong and X. Y. Zhou, Stochastic Controls: Hamiltonian Systems and HJB Equations, vol. 43, Springer, New York, NY, USA, 1999.
[19] S. Peng, "Backward stochastic differential equations and applications to optimal control," Applied Mathematics and Optimization, vol. 27, no. 2, pp. 125-144, 1993. 


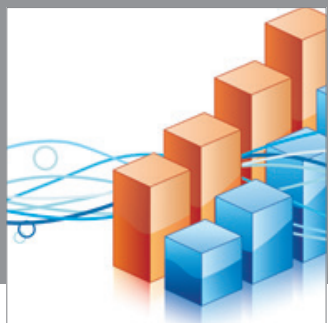

Advances in

Operations Research

mansans

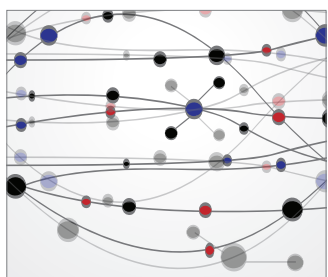

The Scientific World Journal
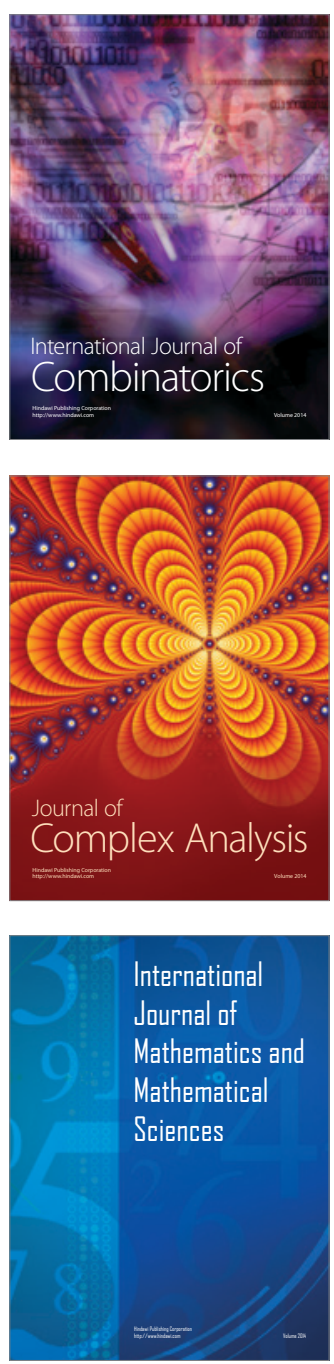
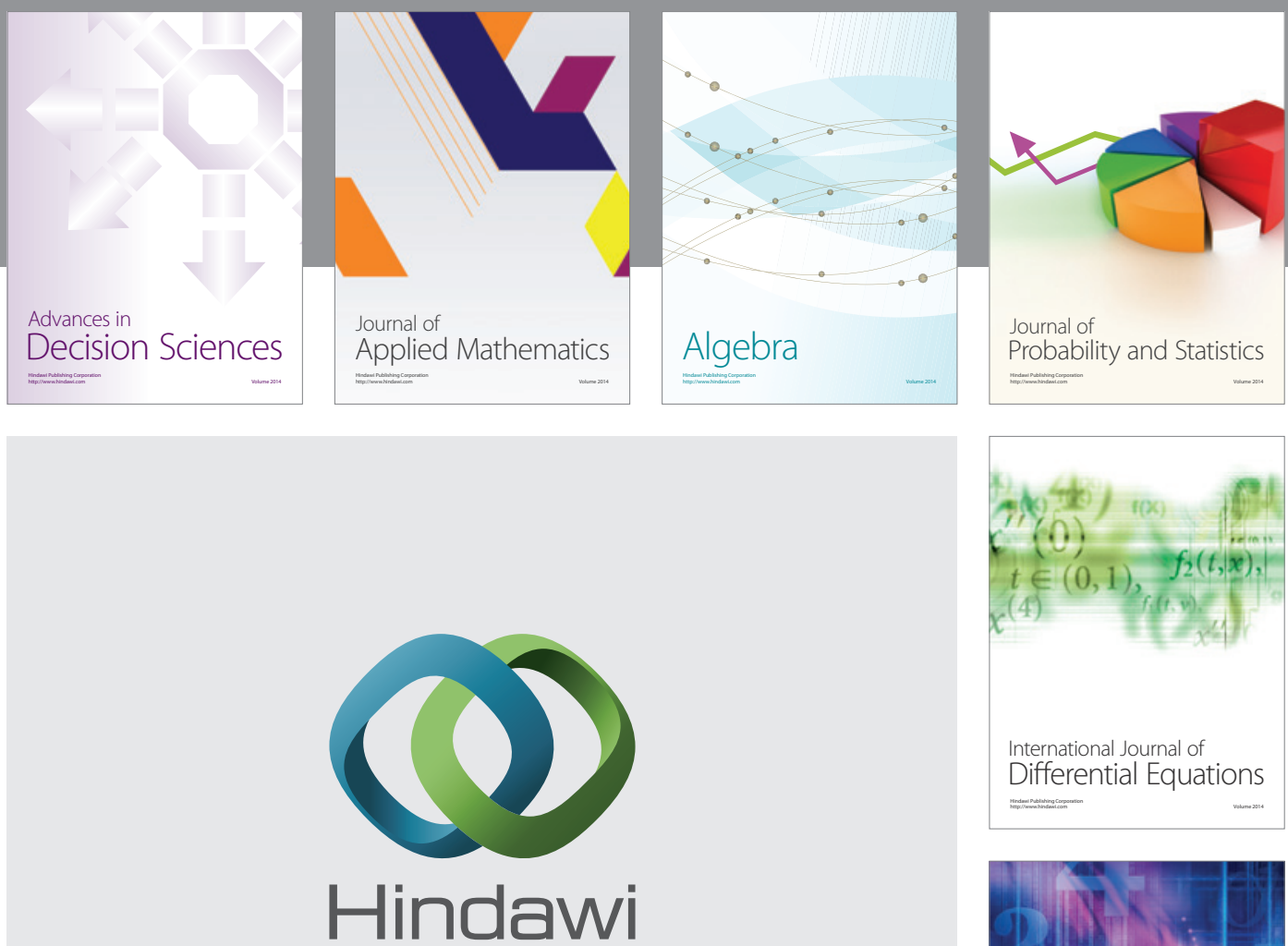

Submit your manuscripts at http://www.hindawi.com
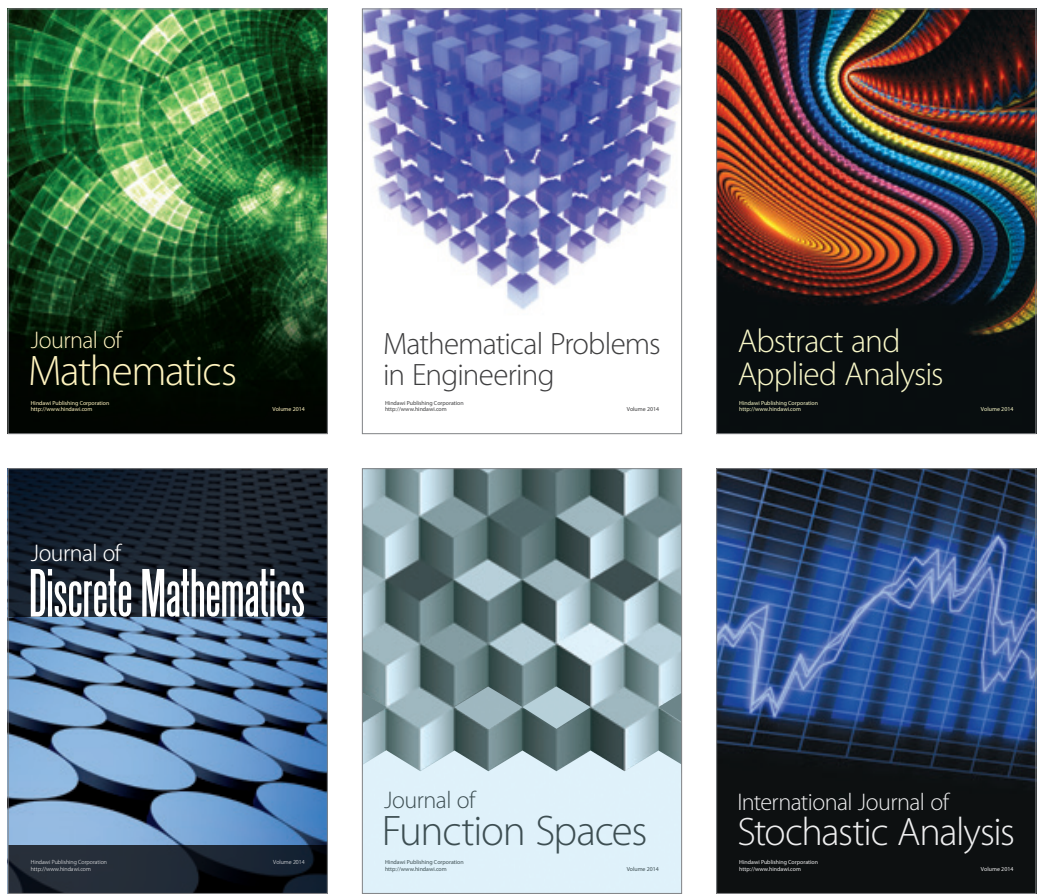

Journal of

Function Spaces

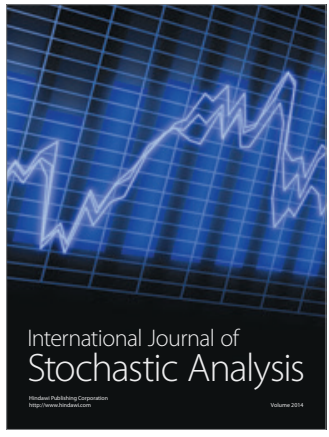

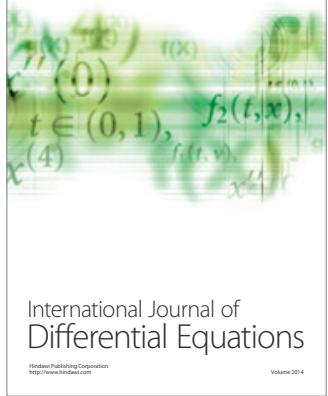
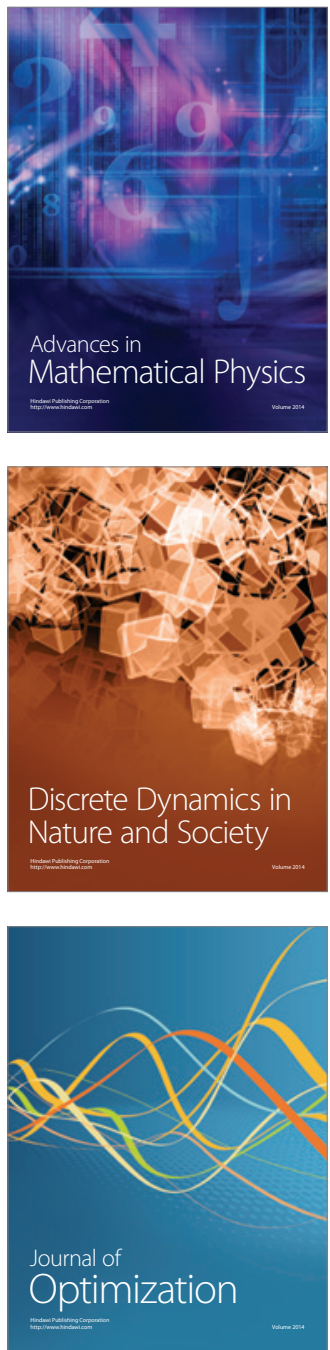